\title{
Towards Automatic Learning in LVCSR: Rapid Development of a Persian Broadcast Transcription System
}

\author{
Christian Gollan and Hermann Ney \\ Human Language Technology and Pattern Recognition - Computer Science Department \\ RWTH Aachen University, D-52056 Aachen, Germany \\ \{gollan, ney\}acs.rwth-aachen. de
}

\begin{abstract}
We present a new method for automatic learning and refining of pronunciations for large vocabulary continuous speech recognition which starts from a small amount of transcribed data and uses automatic transcription techniques for additional untranscribed speech data.

The recognition performance of speech recognition systems usually depends on the available amount and quality of the transcribed training data. The creation of such data is a costly and tedious process and the approach presented here allows training with small amounts of annotated data.

The model parameters of a statistical joint-multigram grapheme-to-phoneme converter are iteratively estimated using small amounts of manual and relatively larger amounts of automatic transcriptions and thus the system improves itself in an unsupervised manner.

Using the new approach, we create a Persian broadcast transcription system from less than five hours of transcribed speech and 52 hours of untranscribed audio data.
\end{abstract}

Index Terms: Automatic Learning, Unsupervised Training, Dictionary Learning, Automatic Transcription

\section{Introduction}

The recognition performance of a state-of-the-art large vocabulary continuous speech recognition (LVCSR) system depends on the amount and quality of task representative training data. Huge collections of language resources are necessary for robust estimation of the model parameters. The current version of the Arabic broadcast transcription system - described in [1] - is trained on more than 1000 hours of speech data and more than 1 billion running words of text data. The data collection process is still ongoing to improve the systems transcription performance by increasing the amount of training data.

Often, only a relatively small amount of annotated speech data is available when an LVCSR system is build for a new language. In general, whenever a new system is designed, task representative speech data needs to be collected and manually transcribed which is a time-consuming and costly process. Commonly, an annotated speech corpus and a pronunciation dictionary are used for acoustic model training. For language model training, large text collections are used - most often online newspaper archives. The creation of the pronunciation dictionary and manual transcription of speech are by far the most expensive steps in setting up a new ASR system.

Using the automatic transcriptions of speech data to train an ASR system is commonly referred to as unsupervised training [2]. Automatic annotations are not only cheaper than manual annotations but can also be obtained much faster. On the downside, automatic annotations commonly contain more transcription errors than manually transcribed ones. Especially

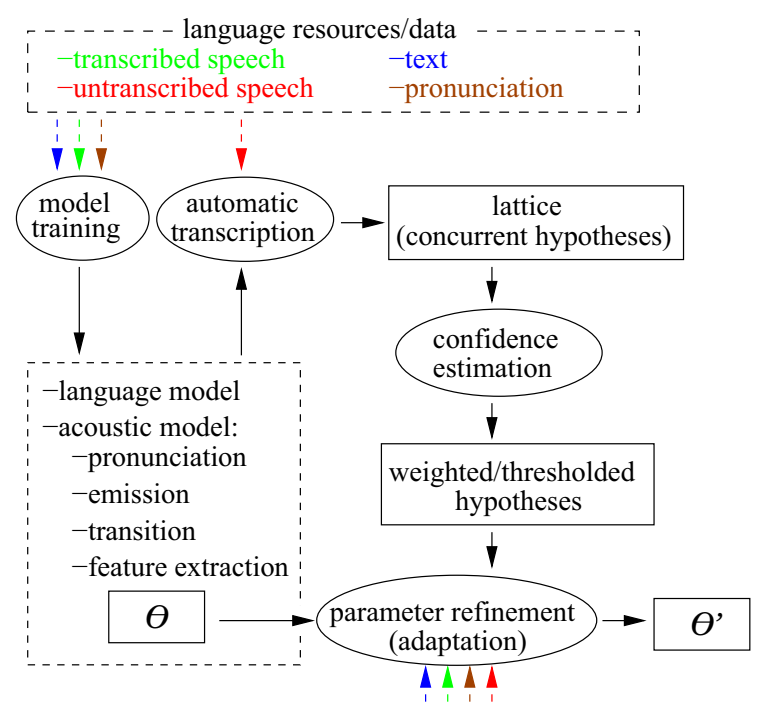

Figure 1: Initial setup in the Automatic Learning framework: First iteration of unsupervised/automatic parameter refinement.

when the ASR system itself is trained only on a small amount of transcribed speech data. To avoid problems due to this drawback, confidence scores can be applied to remove the most likely errors from the automatic annotations. Here, we use the term automatic learning, to describe the ability of an ASR system to improve its parameters in general without a manually annotated speech corpus. Ideally, such an automatic transcription system trains and improves its model parameters without any human setup efforts. A broadcast news transcription system could improve its performance online by collecting broadcast audio streams and internet text news to automatically reestimate its model parameters.

Figure 1 shows an iteration of the automatic learning concept for an ASR system. Well known as acoustic model adaptation is the iterative improvement of the emission model parameters using the previous automatic transcriptions of the recognition task. If we use large amounts of additional speech data, we call the process unsupervised training. Depending on the amount and the quality of the automatic transcriptions, different acoustic refinement methods are used, e.g., linear transform based adaption, maximum a posteriori adaptation or full BaumWelch re-estimation.

In the automatic learning framework also the parameters of the language or the acoustic feature generation model can be iteratively improved. E.g. the parameters of a multi-layer perceptron (MLP) network can be refined using additional automatically transcribed data. 
Table 1: Persian words with automatically refined pronunciations.

\begin{tabular}{|c|c|c|c|c|}
\hline $\begin{array}{l}\text { word } \\
\text { rank }\end{array}$ & word & $\begin{array}{c}\text { ASCII } \\
\text { transliteration } \\
\end{array}$ & $\begin{array}{c}\text { first 2-best } \\
\text { pronunciations }\end{array}$ & $\begin{array}{c}\text { pron. } \\
\text { probab. }\end{array}$ \\
\hline 1 & 9 & W & $\begin{array}{c}\mathrm{v} \mathrm{A} \\
\mathrm{O}\end{array}$ & $\begin{array}{l}0.65 \\
0.32\end{array}$ \\
\hline 19 & ابر ان & AyrAn & Qiran & 1.0 \\
\hline 90 & استفاده & AstfAdh & $\begin{array}{l}\text { sdfade } \\
\text { Afade }\end{array}$ & $\begin{array}{l}0.92 \\
0.05\end{array}$ \\
\hline 166 & اجثماعي & AjtmAEy & $\begin{array}{l}\text { A S t A m o Q i } \\
\text { A s t A m o Q i }\end{array}$ & $\begin{array}{l}0.91 \\
0.07\end{array}$ \\
\hline 7655 & اوباما & AwbAmA & $\mid \begin{array}{c}\text { Q o b a m a } \\
\text { Q ob } \quad \text { A m m a }\end{array}$ & $\begin{array}{l}0.76 \\
0.09\end{array}$ \\
\hline
\end{tabular}

In this paper, we describe the rapid development of an LVCSR system for Persian broadcast news where we apply the confidence based parameter refinement method for the emission model and the pronunciation model training. In [3] a similar experiment was presented to set up an American-English broadcast transcription system. The training data was artificially reduced to perform unsupervised acoustic model training as a proof of concept. In [4] we have presented the unsupervised emission model training on state/frame level. In this work, we present the automatic learning framework and we propose a novel method for automatic learning and refinement of a pronunciation dictionary. A related approach for dictionary learning is presented in [5], where the authors use an annotated speech corpus and a phoneme language model.

\section{Task and System Description}

\subsection{Persian Language Details}

In a few countries Persian is spoken as official language, i.e. Iran, Afghanistan and Tajikistan. The language is often referred to as Farsi which is the local name. Further languages are spoken in Iran, e.g. Luri and Bakhtiari. These languages are related to Persian but they have different words and grammar. Modern Iranian Persian is the official language in Iran and is therefore the main language in Iranian broadcasts and newspapers.

As in Arabic, short vowels are generally omitted in Persian documents and only the long vowels are written in the text. In Modern Iranian Persian, a modified variant of the Arabic alphabet is used for writing, i.e., some of the Arabic letters are modified and a few additional letters are used.

Table 1 shows frequent Persian words from our language model text data and their pronunciations. The appearance of Persian characters depends on their context and position within a word and for an untrained person it can be difficult to read them. Therefore, the ASCII transliteration is often used to represent Arabic or Persian text by the latin alphabet.

\subsection{Persian Language Resources}

Usually, huge data collections - in particular annotated speech corpora - are used to build LVCSR systems. Unfortunately, large collections are not available for all languages. For Persian, there are only a few annotated data resources publicly available.

Acoustic data: To train our initial acoustic model we use the the Farsdat speech corpus [6]. This database contains less than 5 hours of annotated read speech of Persian sentences read by 304 speakers. Clearly, this corpus is not the perfect fit to train acoustic models for a broadcast transcription system.

We collected audio recordings - representing our transcription task - from the news channel of the Islamic Republic of Iran News Network (IRINN). The first broadcast recording was collected in February 2008. It was annotated by Persian tran-
Table 2: Text corpora statistics.

\begin{tabular}{|l||c|c|c|c|}
\hline & $\begin{array}{c}\text { \# running } \\
\text { words }\end{array}$ & $\begin{array}{c}\text { \#uniq } \\
\text { words }\end{array}$ & $\begin{array}{c}\text { 50k voc. } \\
\text { OOV [\%] }\end{array}$ & ppl. \\
\hline $\begin{array}{l}\text { Hamshahri corpus [7] } \\
\text { Jun. 1996-Feb. 2003 }\end{array}$ & $64 \mathrm{~m}$ & $531 \mathrm{k}$ & 2.7 & 141.1 \\
\hline $\begin{array}{l}\text { Hamshahri web text } \\
\text { Jun. 2006-Mar. 2008 }\end{array}$ & $26 \mathrm{~m}$ & $222 \mathrm{k}$ & 2.1 & 114.5 \\
\hline $\begin{array}{l}\text { IRIB web text } \\
\text { Oct. 2005-Mar. 2008 }\end{array}$ & $30 \mathrm{~m}$ & $240 \mathrm{k}$ & 3.1 & 45.0 \\
\hline $\begin{array}{l}\text { IRINN web text } \\
\text { Aug. 2006-Mar. 2008 }\end{array}$ & $10 \mathrm{~m}$ & $134 \mathrm{k}$ & 2.5 & 61.6 \\
\hline
\end{tabular}

scribers to be used as development and test set for our system development process. Since then, we have continuously collected more additional audio data from the IRINN satellite broadcasts. The data was decoded from MPEG-1 layer-2 audio and sampled down to $16 \mathrm{kHz}$ for feature extraction.

Text data: For language modeling, we use different news text archives as presented in Table 2. In our first system setup we used the already preprocessed Hamshahri text corpus [7] compiled from the Hamshahri Iranian newspaper online archive. The Hamshahri corpus covers news articles from June 1996 until February 2003. In addition we download Hamshahri web texts as they are being made available. Furthermore, we collected news texts from the Islamic Republic of Iran Broadcasting (IRIB) web site and from the IRINN web site. Persian is a less morphologically complex language than Arabic. We estimate low out-of-vocabulary (OOV) rates using the top 50k words of the complete text compilation.

\subsection{System Description}

The acoustic model of our initial transcription system was trained on a relatively small amount of read speech. We used the manually produced phoneme alignment of the Farsdat corpus to extract a pronunciation dictionary. This pronunciation dictionary was used to train a data driven statistical graphemeto-phoneme (G2P) model [8] to automatically generate pronunciations for the $50 \mathrm{k}$ recognition lexicon. Table 5 presents the statistics of the Farsdat pronunciation dictionary and the final recognition lexicon. We generate $N$-best pronunciation lists using the G2P approach to produce the pronunciation alternatives which are mostly caused by the short vowels.

In the following, we present the system details of the latest system we obtained due to iterative unsupervised training of the emission and pronunciation model parameters.

System details:

- Speaker and domain independent acoustic model

- Speaker adaptation: VTLN + SAT-CMLLR + MLLR

- 45 dimensional acoustic vectors after applying LDA on 9 time consecutive stacked 17 dimensional features (MFCC and voicing feature)

- 3-state left-to-right HMM topology

- 29 phonemes +2 noise models + silence state

- 4038 decision tree tied within-word triphone states

- $129 \mathrm{k}$ densities within Gaussian mixture models with globally pooled diagonal covariance

- Maximum likelihood training using Viterbi approximation

- $50 \mathrm{k}$ recognition vocabulary

- 4-gram language model with 28 million $M$-grams

The main difference to our initial system is the number of emission model parameters. Due to the relatively small Farsdat speech corpus, we estimated $3 \mathrm{k}$ densities for 1517 states. 


\section{Automatic Learning}

In the automatic learning framework the model parameters of a speech recognition system are iteratively improved for the long term using additional task representative data. Ideally, this data is collected during the application of the system.

\subsection{Automatic Transcription}

In the recognition/speech-decoding process the most likely word sequence $\hat{w}_{1}^{N}$ is determined by Eq. 1 using the Bayes decision rule. The decision depends on the language model probability $p\left(w_{1}^{N}\right)$ and the acoustic probability $p\left(x_{1}^{T} \mid w_{1}^{N}\right) . x_{1}^{T}$ is the acoustic feature vector sequence. The true language probability is approximated by an $M$-gram language model (LM), see Eq. 2. We approximate and decompose the true acoustic probability $p\left(x_{1}^{T} \mid w_{1}^{N}\right)$ into the pronunciation probability $p_{\theta_{\mathrm{p} m}}\left(v_{1}^{N} \mid w_{1}^{N}\right)$, the emission probability $p_{\theta_{\mathrm{em}}}\left(x_{t} \mid s_{t}, v_{1}^{N}\right)$, and the transition probability $p_{\theta_{\mathrm{t} m}}\left(s_{t} \mid s_{t-1}, v_{1}^{N}\right)$, as described in Eq. 4 and Eq. 5, where $v_{1}^{N}$ denotes a pronunciation sequence and $s_{t}$ is an HMM state at time $t$.

The pronunciation scale $\alpha$ and the acoustic scale $\beta$ are used to cope with the model approximations and with different amounts of used training data to model the true probability distributions.

$$
\begin{aligned}
\hat{w}_{1}^{N} & =\underset{w_{1}^{N}}{\operatorname{argmax}}\left\{p\left(x_{1}^{T} \mid w_{1}^{N}\right) \cdot p\left(w_{1}^{N}\right)\right\} \\
p\left(w_{1}^{N}\right) & :=\prod_{n=1}^{N} p_{\theta_{1 m}}\left(w_{n} \mid w_{n-M+1}^{n-1}\right) \\
p\left(x_{1}^{T} \mid w_{1}^{N}\right) & :=\max _{v_{1}^{N}}\left\{p_{\theta_{\mathrm{p} m}}\left(v_{1}^{N} \mid w_{1}^{N}\right)^{\alpha} p_{\theta_{\mathrm{e} m, t m}}\left(x_{1}^{T} \mid v_{1}^{N}\right)^{\beta}\right\} \\
p_{\theta_{\mathrm{p} m}}\left(v_{1}^{N} \mid w_{1}^{N}\right) & :=\prod_{n=1}^{N} p_{\theta_{\mathrm{p} m}}\left(v_{n} \mid w_{n}\right) \\
p_{\theta_{\mathrm{e} m, t m}}\left(x_{1}^{T} \mid v_{1}^{N}\right) & :=\max _{s_{1}^{T} \mid v_{1}^{N}} \prod_{t=1}^{T} p_{\theta_{\mathrm{em}}}\left(x_{t} \mid s_{t}\right) p_{\theta_{\mathrm{t} m}}\left(s_{t} \mid s_{t-1}\right)
\end{aligned}
$$

\subsection{Confidence Scores}

In the speech decoding process different dynamic pruning methods are applied to restrict the list or set of competing word sequences. This list can be efficiently represented using a lattice $L$. With the forward-backward algorithm, we efficiently compute the relation between the competing hypotheses by estimating the lattice link posterior probabilities [9]. Depending on the lattice link labels and structure we can compute the confidence scores for different events, e.g. word, phoneme, state or pronunciation confidence scores.

A pronunciation lattice link $[w, v ; \tau, t] \in L$ consists of a word $w$, a pronunciation $v$, a start time $\tau$ and end time $t$. The lattice link posterior probability is denoted as $p\left([w, v ; \tau, t] \mid x_{1}^{T}\right)$. We now can calculate the pronunciation confidence score $C\left(v, n ; L, \hat{w}_{1}^{N}\right)$ for our first best word sequence $\hat{w}_{1}^{N}$ using the maximum approximation analogously to the maximum word confidence scores as presented in [10]:

$$
C\left(v, n ; L, \hat{w}_{1}^{N}\right):=\max _{\substack{\hat{t}: \\ \tau_{\hat{w}_{n}} \leq \hat{t} \leq t_{\hat{w}_{n}}}} \sum_{\substack{\left[\hat{w}_{n}, v ; \tau^{\prime} ; t^{\prime}\right]: \\ \tau^{\prime} \leq \hat{t}^{\prime} \leq t^{\prime}}} p\left(\left[\hat{w}_{n}, v ; \tau^{\prime}, t^{\prime}\right] \mid x_{1}^{T}\right)
$$

\subsection{Pronunciation Model Refinement}

We are using a statistical data driven pronunciation model derived from the G2P conversion method presented in [8]. There, the joint probability distribution $p(w, v)$ is reduced to the probability distribution $p\left(q_{1}^{J}\right)$ modeled by a standard $M$-gram:

$$
p\left(q_{1}^{J}\right):=\prod_{j=1}^{J} p\left(q_{j} \mid q_{j-M+1}^{j-1}\right)
$$

A graphone sequence $q_{1}^{J}$ corresponds to a word pronunciation pair $(w, v)$. In our experiments a graphone $q$ is a pair of a word-symbol/grapheme and pronunciation-symbol/phoneme, where a grapheme or phoneme can be the empty grapheme or phoneme respectively. $S(w, v)$ defines the set of all possible graphone sequences to segment the pair $(w, v)$ into graphones. We calculate the pronunciation probability $p_{\theta_{\mathrm{G} 2 P}}(v \mid w)$ using the G2P model the following way:

$$
p_{\theta_{\mathrm{G} 2 P}}(v \mid w):=\frac{\max _{q_{1}^{J} \in S(w, v)} p\left(q_{1}^{J}\right)}{\sum_{v^{\prime}} \max _{q_{1}^{\prime} J^{\prime} \in S\left(w, v^{\prime}\right)} p\left(q_{1}^{\prime J^{\prime}}\right)}
$$

The pronunciation counts \# $(v, w)$ and the word counts \# $(w)$ are estimated from the first best transcription alignment path and represent how often these events were observed. We refine the pronunciation probabilities in a maximum a posteriori fashion based on the normalized pronunciation counts and the balancing parameter $\lambda$ :

$$
p_{\theta_{\mathrm{p} m}^{\prime}}(v \mid w):=\frac{\#(v, w)}{\lambda+\#(w)}+\frac{\lambda}{\lambda+\#(w)} p_{\theta_{\mathrm{p} m}}(v \mid w)
$$

For the automatic transcriptions, we apply the pronunciation confidence score to estimate only counts for events with high confidence. This is done for the first best hypotheses $\hat{w}_{1}^{N}$ of the automatic transcriptions:

$$
\#(v, w)_{\hat{w}_{1}^{N}}:=\sum_{\substack{n: \\ C\left(v, n ; L, \hat{w}_{1}^{N}\right)>\operatorname{thr} .}}^{N} \delta\left(w, \hat{w}_{n}\right), \quad \#(w):=\sum_{v} \#(v, w)
$$

\subsection{Emission Model Re-estimation}

We perform unsupervised acoustic model training - more precisely, iterative acoustic emission model re-estimation - using confidence-thresholded automatic transcriptions. For Gaussian mixture training, the data filtering process is done on state/frame level to select the pairs of a state and a acoustic feature vector based on their confidence score. This selection or filtering process is more precise than performing the thresholding on sentence or word level and was successfully applied for unsupervised training [4], as well as for unsupervised acoustic model adaptation [11].

Depending on the purpose we estimate confidence scores for other events. We perform the data selection for the Gaussian mixture training or the LDA estimation based on the confidence scores of tied HMM states. Whereas for the estimation of the state tying, we threshold the observations based on their allophone state confidence scores.

\section{Experiments}

Table 3 summarizes the rapid development of the Persian broadcast transcription system which was started in February 2008. The initial acoustic model was trained on the Farsdat speech corpus resulting in a WER of $75.8 \%$ on the IRINN development set. We use the manually produced transcriptions of the IRINN 
Table 3: Training steps with the corresponding emission model statistics and resulting system performance on the dev set.

\begin{tabular}{|l||c|c|c|c||c|}
\hline \multicolumn{1}{|c|}{$\begin{array}{c}\text { training } \\
\text { step }\end{array}$} & $\begin{array}{c}\text { audio } \\
\text { data }[\mathrm{h}]\end{array}$ & $\begin{array}{c}\text { selected } \\
\text { data }[\mathrm{h}]\end{array}$ & \#states & \#densities & $\begin{array}{c}\text { dev set } \\
\text { WER [\%] }\end{array}$ \\
\hline \hline Farsdat & 4.7 & 4.7 & 1517 & $3 \mathrm{k}$ & 75.8 \\
\hline \hline $1 . \theta_{\mathrm{e} m}$ & 11.4 & 8.1 & 1517 & $6 \mathrm{k}$ & 72.7 \\
\hline $2 . \theta_{\mathrm{e} m}$ & 11.4 & 8.6 & 1517 & $12 \mathrm{k}$ & 69.8 \\
\hline $3 . \theta_{\mathrm{e} m}$ & 23.6 & 15.8 & 1517 & $48 \mathrm{k}$ & 63.7 \\
\hline $4 . \theta_{\mathrm{e} m} / \theta_{\mathrm{lm}}, \theta_{\mathrm{p} m}$ & 23.6 & 16.6 & 2281 & $72 \mathrm{k}$ & $61.1 / 57.3$ \\
\hline $5 . \theta_{\mathrm{e} m}$ & 39.0 & 29.1 & 2281 & $144 \mathrm{k}$ & 51.7 \\
\hline $6 . \theta_{\mathrm{e} m}$ & 39.0 & 29.1 & 3322 & $205 \mathrm{k}$ & 51.4 \\
\hline $7 . \theta_{\mathrm{e} m} / \theta_{\mathrm{p} m}$ & 39.0 & 30.2 & 3322 & $207 \mathrm{k}$ & $50.7 / 50.2$ \\
\hline $8 . \theta_{\mathrm{e} m}$ & 52.0 & 39.6 & 3322 & $209 \mathrm{k}$ & 48.0 \\
\hline $9 . \theta_{\mathrm{e} m}$ & 52.0 & 39.1 & 4038 & $247 \mathrm{k}$ & 46.5 \\
\hline $10 . \theta_{\mathrm{e} m} / \theta_{\mathrm{p} m}$ & 52.0 & 41.4 & 4038 & $249 \mathrm{k}$ & $45.9 / 45.1$ \\
\hline $11 . \theta_{\mathrm{e} m} / \theta_{\mathrm{p} m}$ & 52.0 & 41.0 & 4038 & $129 \mathrm{k}$ & $44.1 / 43.4$ \\
\hline $12 . \theta_{\mathrm{e} m}$ & 52.0 & 40.0 & 4038 & $129 \mathrm{k}$ & 42.3 \\
\hline
\end{tabular}

Table 4: Speech corpora statistics.

\begin{tabular}{|l||c|c|c|c|}
\hline & Farsdat & AT & dev & test \\
\hline \hline data [h] & 4.7 & 52.0 & 0.3 & 2.5 \\
\hline \# running words & $41 \mathrm{k}$ & $390 \mathrm{k}$ & $3 \mathrm{k}$ & $18 \mathrm{k}$ \\
\hline \# segments & $6 \mathrm{k}$ & $19 \mathrm{k}$ & 116 & 890 \\
\hline \# speaker cluster & 304 & 1111 & 11 & 36 \\
\hline OOV [\%] & 2.0 & - & 2.6 & 3.4 \\
\hline ppl. & 225.0 & 156.9 & 141.1 & 201.5 \\
\hline
\end{tabular}

broadcast recordings from February 2008 as evaluation sets. For unsupervised training, we have collected further IRINN recordings since March 2008 and the automatic transcription (AT) data set increased over time up to 52 hours. Table 4 gives an overview of the currently used speech corpora.

The initial G2P model was trained on the Farsdat pronunciation dictionary. With this initial G2P model, we generated the 4-best pronunciations for the words of our 50k recognition vocabulary. In the later unsupervised training iterations we estimated the pronunciation counts on the manual and the automatic transcriptions to refine the pronunciation probabilities of the training and the recognition lexicon. Furthermore, we re-estimated the G2P model based on the weighted pronunciation counts. Then, we updated the pronunciation entries of the recognition lexicon based on the 4-best pronunciations generated by the updated G2P model.

The improvements due to the model updates are presented in Table 3. Until the 3rd training step, we re-estimated the Gaussian mixture model using additional automatic transcriptions which allowed us to increase the number of densities and the WER dropped to $63.7 \%$. In the 4th training step we have re-estimated the CART tying as well as the LDA matrix leading to a WER of $61.1 \%$. Furthermore, using the same emission model in the 4th training step with an improved LM and a refined PM, we measured the WER of $57.3 \%$. Currently, we are in the 12 th training step leading to a WER of $42.3 \%$ with a speaker independent acoustic model. Table 6 presents the results of our 3-pass Persian transcription system.

As can be seen, most of the gain is due to the increased parameter set but the estimation of these is only robust using the additional automatic transcribed training data.

\section{Conclusion and Outlook}

We presented the rapid development of a Persian broadcast transcription system using a relatively small speech database. Within 6 weeks, we set up an LVCSR system using less than 5 hours of manual transcriptions and a manually created pronunciation dictionary covering only 1000 words. We have success-
Table 5: Dictionary statistics.

\begin{tabular}{|c|c|c|c|c|c|}
\hline & & & \\
\hline & Farsdat & recog. & & $\mid \mathrm{dev}$ & test \\
\hline \# words & 1017 & 499939 & spk. indep. & || 42.3 & 447.9 \\
\hline \# pronunciations & 4853 & 190168 & SAT-CMLLR & 38.5 & 41.9 \\
\hline \# homophones & 226 & 12861 & MLLR & $\mid 37.5$ & 39.9 \\
\hline
\end{tabular}

fully applied the automatic learning framework to re-estimate the emission model parameters and the pronunciation model parameters in an unsupervised training fashion.

In future work we will integrate acoustic MLP features into our transcription system. The MLP network can then also be iteratively re-estimated based on the automatic transcriptions and their phoneme confidence scores. Furthermore, we will try to perform language model adaptation using large amounts of automatic transcriptions to improve the systems performance for the long term.

Our future plans include the investigation of the amount of transcribed training data required to set up an ASR system. Ideally, it would be possible to bootstrap systems without any transcribed speech data at all.

Acknowledgement. This work was partly supported by Applications Technology, Inc. (AppTek).

\section{References}

[1] D. Rybach, S. Hahn, C. Gollan, R. Schlüter, and H. Ney, "Advances in Arabic Broadcast News Transcription at RWTH," in Proc. IEEE Automatic Speech Recognition and Understanding Workshop, Kyoto, Japan, Dec. 2007, pp. 449-454.

[2] G. Zavaliagkos and T. Colthurst, "Utilizing Untranscribed Training Data to Improve Performance," in DARPA Broadcast News Transcription and Understanding Workshop, Landsdowne, VA, USA, Feb. 1998, pp. $301-305$.

[3] F. Wessel and H. Ney, "Unsupervised Training of Acoustic Models for Large Vocabulary Continuous Speech Recognition," IEEE Transactions on Speech and Audio Processing, vol. 13, no. 1, pp. 23 - 31, Jan. 2005.

[4] C. Gollan, S. Hahn, R. Schlüter, and H. Ney, "An Improved Method for Unsupervised Training of LVCSR Systems," in Proc. European Conf. on Speech Communication and Technology, Antwerp, Belgium, Aug. 2007, pp. 2101-2104.

[5] T. Sloboda and A. Waibel, "Dictionary Learning for Spontaneous Speech Recognition," in Proc. Int. Conf. on Spoken Language Processing, Philadelphia, PA, USA, Oct. 1996, pp. 2328-2331.

[6] M. Bijankhan, J. Sheikhzadegan, M. Roohani, Y. Samareh, K. Lucass, and M. Tabiani, "FARSDAT-The Speech Database of Farsi Spoken Language," in Fifth Australian International Conference on Speech Science and Technology (SST-94), Perth, Australia, Dec. 1994, pp. 826-831.

[7] F. Oroumchian, E. Darrudi, and M. Hejazi, "Assessment of a Modern Farsi Corpus," in 2nd Workshop on Information Technology and its Disciplines (WITID), Kish Island, Iran, Feb. 2004.

[8] M. Bisani and H. Ney, "Investigations on Joint-Multigram Models for Grapheme-to-Phoneme Conversion," in Proc. Int. Conf. on Spoken Language Processing, Denver, CO, USA, Sep. 2002, pp. 105-108.

[9] G. Evermann and P. Woodland, "Large Vocabulary Decoding and Confidence Estimation using Word Posterior Probabilities," in Proc. IEEE Int. Conf. on Acoustics, Speech, and Signal Processing, Istanbul, Turkey, Jun. 2000, pp. 1655 - 1658.

[10] F. Wessel, K. Macherey, and H. Ney, "A Comparison of Word Graph and N-Best List Based Confidence Measures," in Proc. European Conf. on Speech Communication and Technology, Budapest, Hungary, Sep. 1999, pp. 315-318.

[11] C. Gollan and M. Bacchiani, "Confidence Scores for Acoustic Model Adaptation," in Proc. IEEE Int. Conf. on Acoustics, Speech, and Signal Processing, Las Vegas, NV, USA, Apr. 2008, pp. 4289-4292. 